\title{
Making Sense of Coulomb Explosion Imaging
}

\author{
Itamar Luzon, ${ }^{\dagger}$, Ester Livshits, ${ }^{\dagger}, \mathbb{\Upsilon}$ Krishnendu Gope, ${ }^{\dagger}$ Roi Baer, ${ }^{* \dagger}$ and Daniel \\ Strasser ${ }^{*, \dagger}$
}

$\dagger$ Institute of Chemistry, The Hebrew University of Jerusalem, Jerusalem 91904, Israel. $\ddagger$ Fritz Haber Center for Molecular Dynamics and the Institute of Chemistry, The Hebrew University of Jerusalem, Jerusalem 91904, Israel

T These authors contributed equally

E-mail: roi.baer@huji.ac.il; strasser@huji.ac.il

\begin{abstract}
A multifaceted agreement between ab initio theoretical predictions and experimental measurements, including branching ratios, channel-specific kinetic energy release, and three-body momentum correlation spectra, leads to the identification of new mechanisms in Coulomb-explosion (CE) induced two- and three-body breakup processes in methanol. These identified mechanisms include direct nonadiabatic Coulomb explosion responsible for $\mathrm{CO}$ bond-breaking, a long-range "inverse harpooning" dominating the production of $\mathrm{H}_{2}^{+}+\mathrm{HCOH}^{+}$, a transient proton migration leading to surprising energy partitioning in three body fragmentation and other complex dynamics forming products such as $\mathrm{H}_{2} \mathrm{O}^{+}$and $\mathrm{H}_{3}^{+}$. These mechanisms provide general concepts that should be useful for analyzing future time-resolved Coulomb explosion imaging of methanol as well as other molecular systems. These advances are enabled by a combination of recently-developed experimental and computational techniques, using weak ultrafast EUV pulses to initiate the CE and a high-level quantum chemistry approach to follow the resulting field-free non-adiabatic molecular dynamics.
\end{abstract}




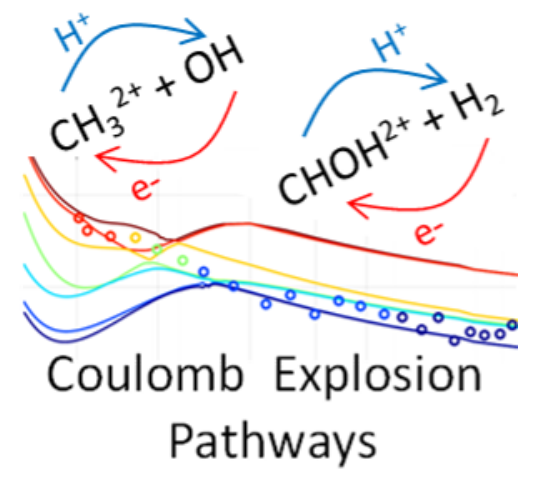

Time-resolved imaging of dynamical events in complex molecular processes is a grand challenge of ultrafast spectroscopy still beyond our reach. One of the ways for achieving this goal involves the use of ultrafast laser pulses to induce a violent Coulomb explosion $(\mathrm{CE})$ in a molecule and then to measure by coincidence-imaging techniques the correlated momenta of the ejected cations ${ }^{1-3}$. It was assumed that the measured momentum correlations could be interpreted in sufficient detail and reliability to allow determination of the instantaneous molecular structure prevailing just before the split-femtosecond occurrence of the explosion ${ }^{4,5}$. However, despite significant advances in the CE technique, interpretation of Coulomb explosion imaging (CEI) data is still limited. Ideally, one would like to have a "button" pressed at zero time which instantaneously pumps sufficient energy into the molecular system for Coulomb-explosion to occur simetime later. One would then be able to use molecular dynamics to analyze the ensuing "trajectories" thereby allowing a detailed interpretation of the experiment. However, meeting this ideal is complicated because typical CEI experiments use low-frequency high-intensity laser pulses to ignite the explosion. These pulses die out eventually but during their operation the molecule absorbs their energy through complex multiphoton processes, and by undergoing complicated field-dressed multi-electron molecular dynamics, including structural rearrangements on the field-dressed states $^{6,7}$, molecular alignment, and bond softening ${ }^{8}$. It is impossible to follow these nonlinear effects using existing computational algorithms, except in very small molecules such as $\mathrm{H}_{2}$ or $D_{2}{ }^{9-12}$. Hence, by the time the pulses have died off and field-free dynamics finally takes over, the molecular configuration has changed too much to allow for a reliable and detailed 
interpretation of the results.

But the problem is not only experimental, it is also computational. Even under fieldfree conditions, the electron-nuclear dynamics is an exceptionally complicated process in polyatomic systems due to their inherent multi-reference character, which complicates the use of single-reference approaches such as time-dependent density functional theory ${ }^{13,14}$ green's function methods ${ }^{15}$ and coupled-cluster approaches ${ }^{16-18}$. Complications remain even when using complete active space multi-configurational self-consistent field ${ }^{19}$ theory due to the inaccurate treatment of dynamical electron correlation in this approach. The combination of CASSCF with $2^{n d}$-order perturbation theory, dubbed CASPT $2{ }^{20}$ seems to overcome all these problems, but wasn't used for dynamics since until recently, since energy and wave function gradients (force and non-adiabatic couplings) weren't implemented due to complications in deriving error-free theoretical expressions for them.

In this article, using a novel combination of experimental and computational approaches, we present a breakthrough development towards time-resolved CEI, addressing both of the above-described challenges. The first element in the combination is the use of high-order harmonic generation $(\mathrm{HHG})^{21-24}$ to produce ultrafast EUV pulses inducing instantaneous single-photon double ionization. ${ }^{25}$ Thus dications are formed in the Franck-Condon (FC) geometry of its time resolved neutral parent. ${ }^{26,27}$ The second element is the use of highly accurate non-adiabatic ab initio molecular dynamics (NA-AIMD) method for interpretation of the experimental results. This development relies on the recently developed CASPT2 gradients, which is unique in the BAGEL code. ${ }^{28,29}$ To demonstrate our novel method we applied it to the doubly-ionized methanol, recently studied in the context of CEI by several groups $^{26,30,31}$. While intense laser CEI of methanol was shown to depend on the exact laser parameters, ${ }^{7}$ we discovered that the single-photon approach allows for a remarkable matching between the experimental and $a b$ initio product branching ratios and kinetic energy release (KER) spectra. In particular, theory-inspired experiments allow for the first time to directly compare detailed experimental and ab initio momenta correlations in three-body di- 
cation breakup. Moreover, the availability of NA-AIMD trajectories offers an unprecedented detailed interpretation of a variety of non-adiabatic processes leading to the observed twoand three-body CE product distributions.
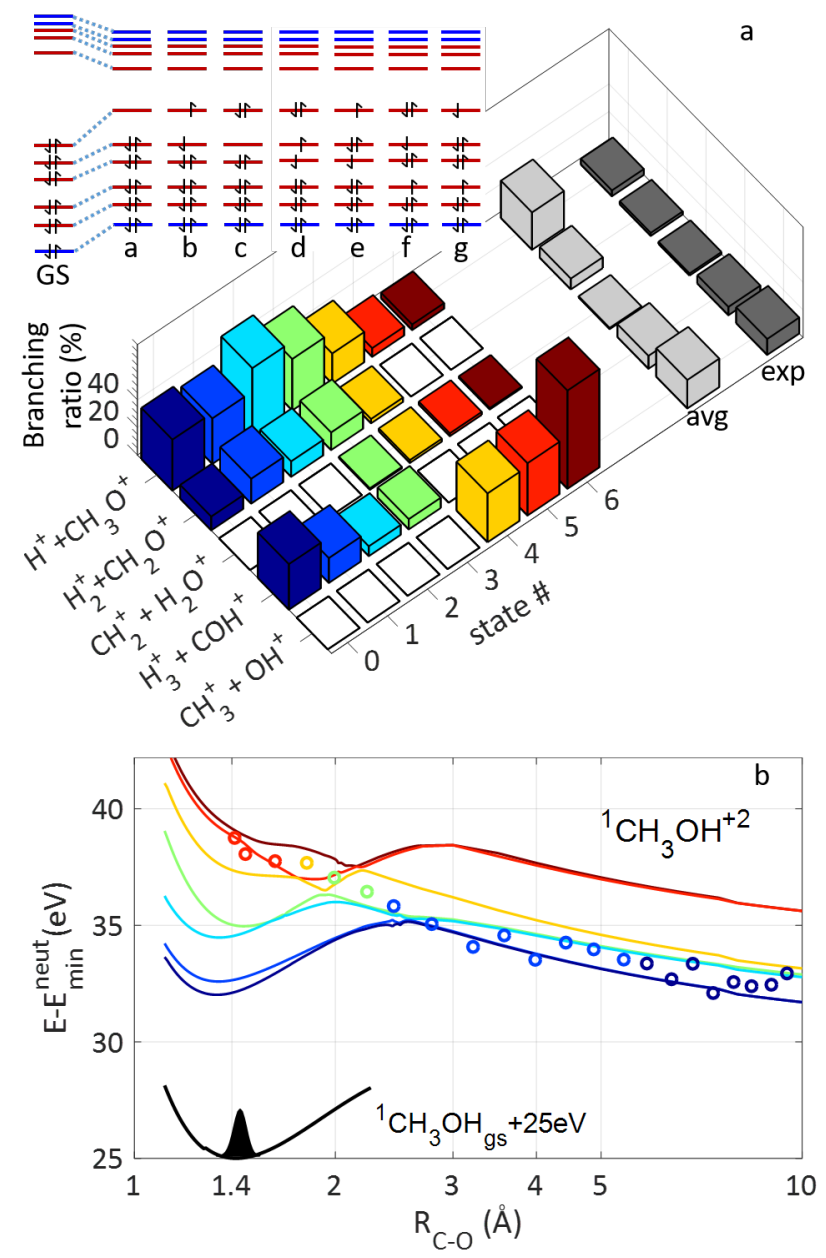

Figure 1: (a) Experimental branching ratios for the two-body CE channels (exp) are compared to the calculated ratios (avg). The average is taken over the product distributions obtained from $\sim 700$ trajectories starting from the 7 lowest adiabatic dication states (numbered 0-6). Inset describes the electronic diabatic configurations dominating the neutral ground state and the 7 lowest adiabatic dication states (see text for details). (b) The adiabatic potential curves for the $\mathrm{CO}$ bond-breaking channel $\mathrm{CH}_{3} \mathrm{OH}^{2+} \rightarrow \mathrm{CH}_{3}^{+}+\mathrm{OH}^{+}$. The empty circles depict the energies and bond lengths along a typical $\mathrm{CO}$ bond-breaking non-adiabatic trajectory at $5.1 \mathrm{fs}$ intervals.

The experimental measurements yield asymptotic cation momenta and branching ratios, which give the relative yields of both two- and three-body CE channels. Five two-body CE channels are observed and their measured branching ratios are compared to ab initio 
predicted yields in Fig. 1. The predominant two-body channels in the experimental data are the $\mathrm{CO}$ bond-breaking $\mathrm{CH}_{3}^{+}+\mathrm{OH}^{+}$and the trihydrogen cation ejection $\mathrm{H}_{3}^{+}+\mathrm{COH}^{+}$ . Proton or $\mathrm{H}_{2}^{+}$ejection, as well as the intriguing proton migration channel producing $\mathrm{CH}_{2}^{+}+\mathrm{H}_{2} \mathrm{O}^{+}$,exhibit lesser yields.

Theoretically, the seven lowest adiabatic dication states are considered, these are dominated by the diabatic configurations corresponding to $2 \mathrm{e}$ removal from the neutral ground state, shown in Fig. 1(a) inset. Adiabatic states 0,1 and 2 are dominated by diabatic configurations $a, b$ and $c$ respectively; state 3 is mainly a linear combination of $c$ and $d$ and linear combinations of configurations $d-g$ form state 4-6. In the FC region, Mulliken charges of the Methyl and Hydroxyl groups of these dication states are about $+1.5 \mathrm{e}$ and $+0.5 \mathrm{e}$ respectively. Therefore, for CO bond-breaking CE channel to occur, a $+0.5 \mathrm{e}$ charge-transfer that forms a potential barrier must take place. As shown in Fig. 1(b), the barrier appears at $R_{C O} \sim 2.8 \AA$ in all seven adiabatic states. Nevertheless, a dication initiated on one of the high excited states can break the CO bond via a series of non-adiabatic transitions. The open circles in Fig. 1(b) show 5.1 fs time steps of a typical CO bond-breaking trajectory, which is initiated on the $5^{\text {th }}$ excited state, rapidly reaching the dication ground state. The required charge-transfer can be also mediated by proton migration, similarly occurring on the high excited states. On the other hand, for states $0-3$ in which $\mathrm{CO}$ bond-breaking is effectively blocked, more complex CE dynamics lead to formation of the trihydrogen $\mathrm{H}_{3}^{+}$cation. Thus, even single-photon $\mathrm{CE}$ requires considering dynamics on a wide range of excited states. As shown in Fig. 1(a) the average computed branching ratios of these three channels exhibit quantitative agreement with the experimental data. However, ab initio-predicted yields of the proton and $\mathrm{H}_{2}^{+}$ejection channels overestimate the final experimental branching ratios.

To obtain mechanistic insight and critically test ab initio computational models it is important to directly compare not only branching ratios but also dynamical observables, such as channel resolved KER spectra. The left column panels of Fig. 2 show the inter-fragment velocity, time derivative of the inter-fragment distance, computed as a function of time 


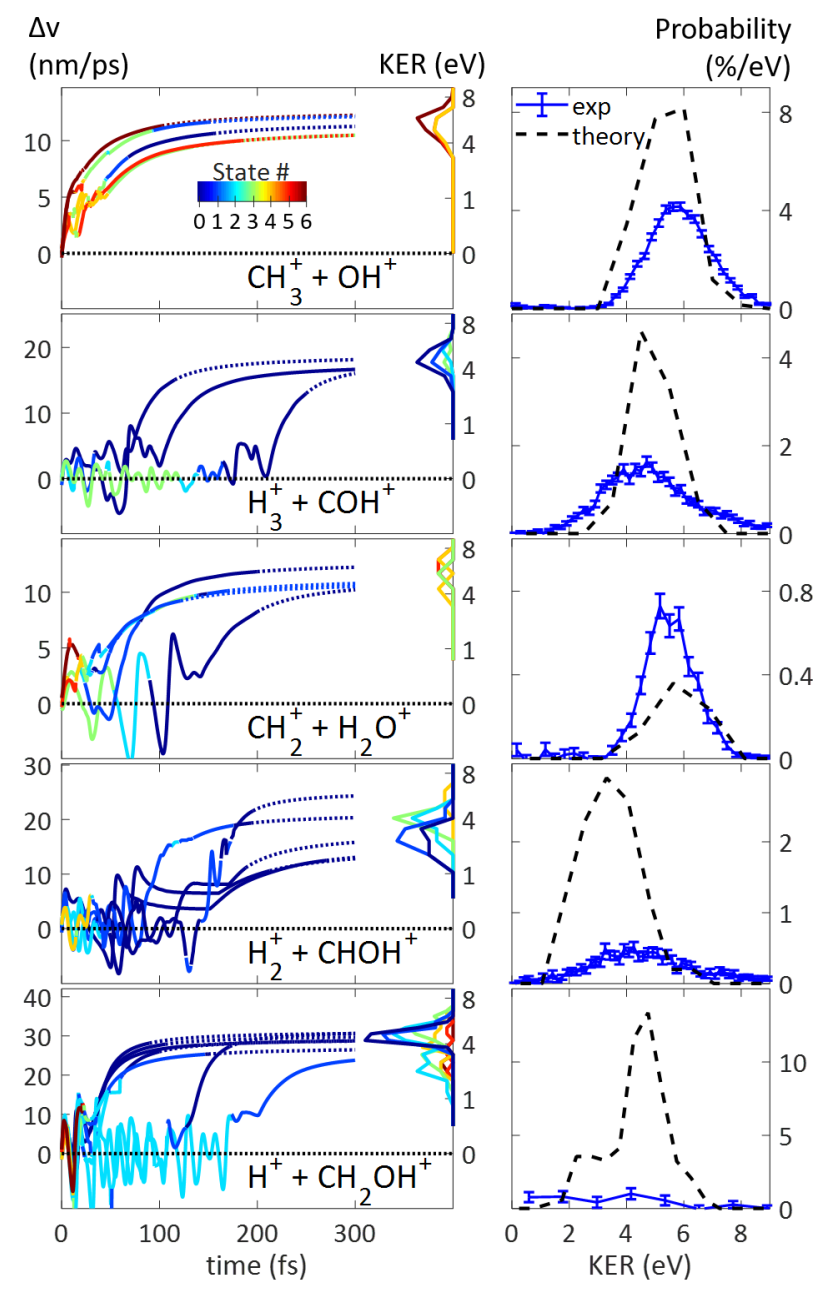

Figure 2: Left column panels: inter-fragment velocities in typical NA-AIMD trajectories as a function of time for different 2-body CE channels. The instantaneous state along each trajectory is color coded, reflecting surface hopping during the time evolution. The KER distributions for different initial states are projected on the right axis. Right column panels: quantitative comparison of experimental KER spectra with the average calculated results. 
for selected trajectories, reflecting the different dynamics leading to the five two-body CE channels. The instantaneous electronic state is encoded in the color. Where trajectory color changes as a function of time evolution represent surface hopping events. For each channel, the asymptotic inter-fragment velocities are used to derive the KER spectra. Within the statistics of the computed trajectories, different initial electronic states exhibit similar KER distributions as can be seen from the KER spectra that are color labeled by the simulated initial electronic excitation. Thus, significant few $\mathrm{eV}$ differences in the initial electronic excitation are largely transferred to product internal electronic as well as ro-vibrational excitation rather than to the KER. The right column panels of Fig. 2 quantitatively compare the average computed KER spectra with the measured data for the five possible two-body CE channels, normalized to the total double ionization yield.

$\mathrm{CH}_{3}^{+}+\mathrm{OH}^{+}$trajectories show strictly positive inter-fragment velocities, hence indicating a direct $\mathrm{CE}$, on a $\sim 40$ fs time scale, facilitated by the strong adiabatic coupling. Returning to the KER distribution of the CO bond-breaking channel, average theoretical and experimental spectra in Fig. 2, show large degree of agreement, with both distributions peaking at $\sim 6 e V$. Due to the strong non-adiabatic coupling discussed earlier, the dissociation occurs on a diabatic potential that is highlighted by the potential along a typical NA-AIMD trajectory shown by open circles in Fig. 1(b). In such prompt dissociation on a strongly repulsive potential, the KER can be expected to reflect the initial instantaneous FC geometry. However, as the trajectories simulated here sample only the very narrow FC region, corresponding to the initial experimental temperature of $300 \mathrm{~K}$, it is difficult to report an explicit correlation between KER and initial geometry. CO bond-breaking by proton migration leading to the final $\mathrm{CH}_{2}^{+}+\mathrm{H}_{2} \mathrm{O}^{+}$channel is also initiated on the higher dication states $(3-6)$. Nevertheless, the CE mechanism that transfers a proton from the Methyl to the Hydroxyl group develops on a longer time scale as indicated by the oscillating inter-fragment velocities. The dissociation time varies, typically dissociating after $50-200 f s$ (see Fig. 2), in which the CO bond completes several vibrations before bond cleavage. Although these longer time 
scales can allow for energy dissipation into internal degrees of freedom, both experimental and theoretical spectra peak at high $\sim 5.5 \mathrm{eV}$, showing once again an impressive agreement.

When the $\mathrm{CO}$ bond does not break, the dication can relax by ejection of a light $\mathrm{H}_{n}^{+}$cation. The highest experimental yield is the $\mathrm{H}_{3}^{+}+\mathrm{COH}^{+}$with both experimental and computed KER distributions peaking at lower $\sim 4.3 \mathrm{eV}$ KER (see Fig. 2). The lower KER can be assigned to the lower initial electronic excitation (states 0-3), as well as to the increased dissipation into the other degrees of freedom as $\mathrm{H}_{3}^{+}$formation requires significant structural rearrangement. This intriguing product channel has been extensively studied using many ionization methods. ${ }^{32,33}$ Similarly to previous AIMD studies on the dication ground state, ${ }^{30,31,33,34}$ typical NA-AIMD trajectories resulting in $\mathrm{H}_{3}^{+}$formationa exhibit a roaming neutral $\mathrm{H}_{2}$ that eventually plucks the last proton connected to $\mathrm{C}$, at which point the vibrationally hot $\mathrm{H}_{3}^{+}$explodes away from the $\mathrm{COH}^{+}$cation. Consequently, the inter-fragment velocity in $\mathrm{H}_{3}^{+}$forming trajectories exhibit extended oscillations before dissociation takes place in agreement with time scales reported by time resolved intense field measurements. ${ }^{30,33,35}$

Returning to the $\mathrm{H}^{+}$and $\mathrm{H}_{2}^{+}$products, we see in Fig. 2 that the simulated trajectories of both channels exhibit significant time delays before dissociation, sometimes up to $250 \mathrm{fs}$. Furthermore, as shown already in Fig. 1(a), the computed proton and $\mathrm{H}_{2}^{+}$ejection trajectories overestimate their quantitative contribution to the coincidence imaging data. Nevertheless, their computed KER spectra are broad, in qualitative agreement with the experimental KER range. In the case of the $\mathrm{CHOH}^{+}+\mathrm{H}_{2}^{+}$channel, the computed time-resolved inter-fragment velocities reveal a remarkable and surprising fragmentation mechanism. In these trajectories, dissociation does not lead immediately to the characteristic rapid acceleration due to the long-range Coulomb repulsion displayed by other channels. CE is preceded by a long $\sim 100$ fs stage of deceleration, reflecting attraction between the nearly separated products. Mulliken charge analysis confirms that at this stage a long range charge transfer occurs. The typical mechanism is as follows: after $\sim 50-100 \mathrm{fs}$, an $\mathrm{H}_{2}^{+}$fragment starts separating and then during the separation, the hole which is located at $\mathrm{H}_{2}^{+}$fragment is back transferred 
to the $\mathrm{CHOH}^{+}$group resulting in deceleration. At this stage both charges are localized on the $\mathrm{CHOH}^{+2}$ fragment that attracts the still separating neutral $\mathrm{H}_{2}$. After $\sim 150$ fs at inter-fragment distances as high as 6 Angstrom, trajectories leading to $\mathrm{CHOH}^{+}+\mathrm{H}_{2}^{+}$undergo a long-range adiabatic charge transfer, clearly reflected in the rapid acceleration by the long-range Coulomb repulsion. This intricate mechanism, which can be described as an "inverse harpooning" mechanism, ${ }^{36}$ results in a broad KER spectrum in both theoretical and experimental results.

The experimental error in the proton ejection channel makes it difficult to compare the shapes of the computed and experimental KER spectra. Nevertheless, the $\mathrm{H}_{2}^{+}$ejection channel data suggests that the low KER events are those missing from the experimental measurement. This, along with the long tail of dissociation delays suggests that sequential dissociation, at times beyond the present calculation, could result in neutral products, which cannot be directly detected in the experiment.

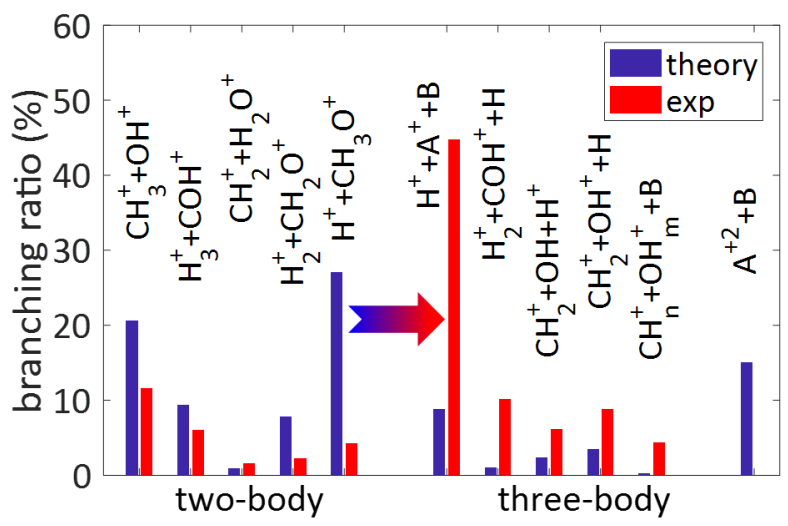

Figure 3: Branching ratios for all 2- and 3-body CE channels, directly comparing theoretical and experimental results.

In search of the theoretically predicted $\mathrm{H}^{+}$and $\mathrm{H}_{2}^{+}$channels, the theoretical result inspired an experimental effort to observe "hidden" neutral products ejected during CE. Fortunately, our present HHG pulse produces vanishing triple-ionization yields. It is therefore possible to assume that all coincident $\mathrm{A}^{+}+\mathrm{B}^{+}$products of methanol for which $m_{A}+m_{B}<32$ amu are accompanied by a "hidden" neutral fragment associated with the missing mass. 
Furthermore, with the advent of high resolution fragment imaging, it is possible to extract also the velocity vector of the "hidden" neutral product, based on the recoil of the center of mass of the "visible" cations. Fig. 3 compares measured and calculated two- and threebody channel branching ratios. Clearly, the missing contributions of computed two-body proton and $\mathrm{H}_{2}^{+}$ejection are found in the experimental excess in three body branching ratios, particularly towards $\mathrm{CHOH}^{+}+\mathrm{H}^{+}+\mathrm{H}_{2}$ and $\mathrm{CHOH}^{+}+\mathrm{H}_{2}^{+}+\mathrm{H}$, which are extremely rare in the computed trajectories. These three-body events that comprise over $50 \%$ of the measured CE data can result from sequential dissociation of electronically excited products indicated by the asymptotic electronic excitation presented in Fig. 2. Furthermore, an additional mechanism for three body $\mathrm{CE}$ could be due to sequential fragmentation of the computed $\sim 16 \%$ neutral $\mathrm{H}$ and $\mathrm{H}_{2}$ ejection from the dication within the 300 fs trajectory time limit.

It is valuable to scrutinize not only the branching ratios but also the three-body correlations of channels that are predicated by the NA-AIMD trajectories. For example CO bond-breaking, accompanied either by ejection of a neutral $\mathrm{H}$ or by a charged proton fragment. Both channels are found with few percent branching ratios in both theoretical and experimental data. We use the Dalitz plot representation of three-body momentum correlations for directly comparing measured experimental data with simulated three-body fragmentation trajectories. Dalitz plot coordinates were originally developed and used to present correlations, beyond the trivial correlations due to energy and momentum conservation, in unimolecular decays into three equal masses. ${ }^{37-39}$ Here, the original coordinates developed by Dalitz are generalized for any three masses, where a random uncorrelated 3-body fragmentation that obey momentum conservation appears as a uniform distribution confined by a unit circle in the $\left(\eta_{1}, \eta_{2}\right)$ plane. ${ }^{37}$ The explicit mass scaled $\eta_{1}, \eta_{2}$ equations are given in the supplementary information (SI).

Figures $4 \mathrm{a}$ and $4 \mathrm{~b}$ show the experimentally measured Dalitz plots for the three-body CO bond-breaking channels, accompanied respectively by ejection of a neutral $\mathrm{H}$ or a proton. The $\eta_{2}$ coordinate is defined as $2 \varepsilon_{H}-1$, where $\varepsilon_{H}$ is the fraction of the total KER carried away 


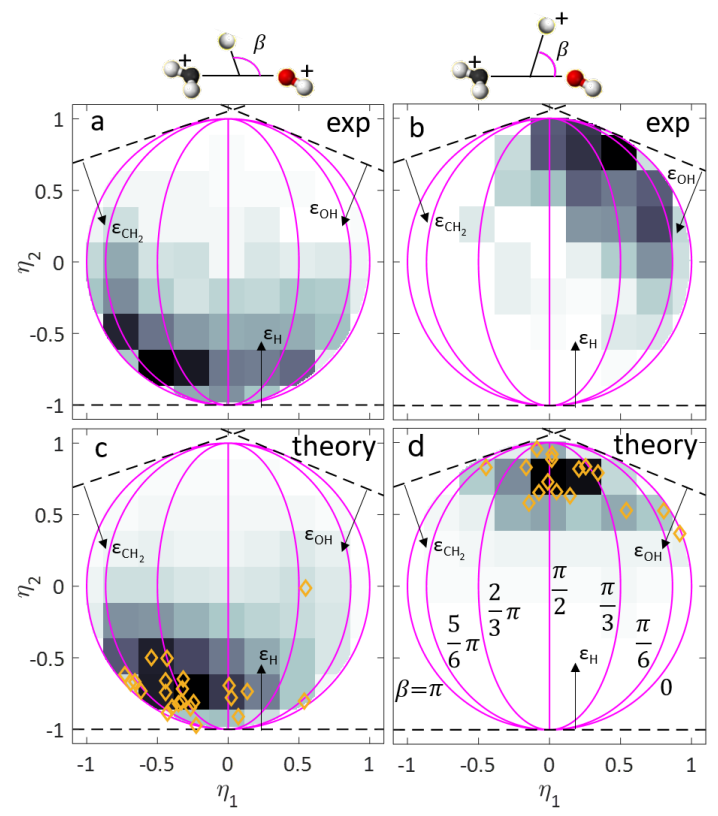

Figure 4: Dalitz plots comparing the experimental (a,b) and ab initio (c,d) 3-body momentum correlations. Left and right panels respectively present the $\mathrm{CH}_{2}^{+}+\mathrm{OH}^{+}+\mathrm{H}$, and $\mathrm{CH}_{2}^{+}+\mathrm{OH}+\mathrm{H}^{+}$channels. Diamond markers indicate individual NA-AIMD trajectories, whilethe gray scale shows the simulated experimental response.

by the $\mathrm{H}$ fragment divided by the maximal fraction allowed by momentum conservation. For convenience, axis corresponding to the KER fractions carried by each fragment are labeled on the Dalitz plots in Fig. 4 as $\varepsilon_{H}, \varepsilon_{O H}$ and $\varepsilon_{C H_{2}}$. The charged proton fragments that exhibit high $\eta_{2}$ values, thus carry higher $\varepsilon_{H}$ compared with neutral $H$ fragments, as can be expected considering the strong Coulombic repulsion. Nevertheless, neutral fragments also exhibit surprising probability to carry away as much as half of the maximal possible kinetic energy, in both cases of neutral $\mathrm{H}$ and $\mathrm{OH}$ fragments. Dalitz plot longitude lines indicate fragmentation geometries with equal Jacobi angle $\beta$ of the $\mathrm{H}$ fragment velocity relative to the $\mathrm{CH}_{2^{-}} \mathrm{OH}$ axis. Where $\beta=0$ and $\beta=\pi$ correspond to a co-linear fragmentation, with $\mathrm{H}$ ejected in the direction of the $\mathrm{OH}$ or $\mathrm{CH}_{2}$ respectively. Neutral $\mathrm{H}$ fragments are observed to be ejected towards the $\mathrm{CH}_{2}^{+}$product, reflecting the methanol ground state geometry. However, proton ejection exhibits an opposite trend that cannot be understood in simple terms of the molecular structure. Clearly, successful theoretical description of three-body correlations is a valuable key for interpretation of CEI measurements. Figures $4 \mathrm{c}$ and $4 \mathrm{~d}$ show the simulated 
Dalitz plots for the $\mathrm{CH}_{2}^{+}+\mathrm{OH}^{+}+\mathrm{H}$ and $\mathrm{CH}_{2}^{+}+\mathrm{OH}+\mathrm{H}^{+}$respectively. Diamond markers indicate individual computed trajectories, while the gray scale distributionis obtained by a Monte-Carlo simulation of the experimental response, assuming isotropic orientation of the dissociation plane. This in agreement with the isotropic angular distributions, measured for all two-body channels. ${ }^{26}$ The remarkable success of the CASPT2 level NA-AIMD trajectory calculations in reproducing the measured three-body correlations allows making sense of the CE dynamics by identifying several underlying mechanisms, for which selected movies are provided in the SI.

For the $\mathrm{CH}_{2}^{+}+\mathrm{OH}^{+}+\mathrm{H}$ channel, typical NA-AIMD trajectories begin with the immediate cleavage of the $\mathrm{CO}$ bond, where ejection of a neutral $\mathrm{H}$ from the methyl cation follows within up to $\sim 250$ fs. These dynamics do not involve significant structural rearrangement, accordingly the measured final momenta correlations reflect the neutral $\mathrm{CH}_{3} \mathrm{OH}$ structure. Computed trajectories with concerted three body breakup within $\sim 70$ fs exhibit particularly fast neutral $\mathrm{H}$ fragments, due to the transient charge transfer between $\mathrm{H}$ and $\mathrm{OH}$ fragments during the dissociation.

For the $\mathrm{CH}_{2}^{+}+\mathrm{OH}+\mathrm{H}^{+}$channel, typical NA-AIMD trajectories exhibit proton motion from the carbon toward the oxygen, resulting in the breaking of both bonds within $~ 100 \mathrm{fs}$. This structural rearrangement of the dication away from the FC geometry, is reflected in the bias towards $\beta \ll \pi / 2$ in both computed and experimental data. Furthermore, NAAIMD trajectories provide mechanistic insight also for CE depositing surprisingly high KER to the neutral $\mathrm{OH}$ fragment: A sequential mechanism of proton migration and dissociation into $\mathrm{CH}_{2}^{+}+\mathrm{H}_{2} \mathrm{O}^{+}$, quickly followed by $\mathrm{H}^{+}$ejection allows transient acceleration of the heavy oxygen mass. Thus transient proton migration results in a counter intuitive KER partitioning between neutral and charged CE products.

CE of polyatomic molecules exhibits rich complex dynamics which interpretation requires successful $a b$ initio description of the underlying mechanisms. The present study demonstrates that single-photon CEI of a polyatomic system can be quantitatively modeled and 
understood by considering non-adiabatic ab initio dynamics on the ground and excited dication states, unperturbed by the low-field ultrafast EUV pulse. The successful description of the measured channel branching ratios, KER spectra and three-body Dalitz plot correlations, allows describing and assigning the underlying CEI mechanisms. CE dynamics exhibit ultrafast transient charge migration, resulting in surprising and non-trivial product correlations. Long-range adiabatic charge exchange or "inverse harpooning" results in a delayed Coulomb explosion of nearly dissociated $\mathrm{H}_{2}+\mathrm{HCOH}^{+2}$ systems. Concerted ejection of neutral and cationic fragments, as well as transient proton migration, result in surprisingly high KER deposited in previously overlooked neutral products of Coulomb explosion. Nevertheless, the presented CASPT2 level NA-AIMD trajectory calculations, uniquely enabled by the BAGEL code, allow making sense of the observed CE dynamics leading to the different final channels. On one hand, final channels such as the prompt CO bond-breaking on a diabatic potential, with or without ejection of a neutral H, can directly reflect the instantaneous structure of the parent neutral species in the observed KER spectra and three body Dalitz plot correlations. On the other hand, branching ratios toward final channels such as $\mathrm{H}_{3}^{+}+\mathrm{COH}^{+}, \mathrm{CH}_{2}^{+}+\mathrm{H}_{2} \mathrm{O}^{+}$, or $\mathrm{CH}_{2}^{+}+\mathrm{OH}+\mathrm{H}^{+}$that involve significant structural rearrangement on the dication can be expected to be sensitive to the parent neutral structure at the instance of single-photon double ionization. The critical comparison between experiment and theory, made possible by the low field single-photon double ionization using ultrafast HHG pulses with CASPT2 level NA-AIMD trajectory computations, opens opportunities for time resolved investigations. In particular of the new surprising mechanisms described in the present study for the relatively simple methanol molecule, but are expected to be ubiquitous in molecular and macro-molecular systems, multiply ionized due to all forms of EUV and X-ray radiation damage..$^{40,41}$

The single-photon CEI experimental setup has been described earlier. ${ }^{26}$ In brief, ultrafast EUV pulses are generated using HHG by a $2.1 \mathrm{~mJ}$ near IR laser pulse, ${ }^{42}$ focused in a semiinfinite neon gas cell. ${ }^{43}$ The low intensity EUV pulses are spatially filtered from the near-IR 
that have higher divergence. ${ }^{44}$ At the center of home build 3D coincidence imaging spectrometer, the EUV pulse ionizes a skimmed effusive beam of methanol, where the cationic products are accelerated towards a time and position sensitive detector. ${ }^{26,45}$ Low count rate and center of mass (CM) momentum conservation are used to suppress random coincidence cation signal due to dissociative ionization of two different parent molecules. Any residual contributions from random coincidence are estimated and subtracted based on the measured single cation event probabilities. Further details of the three-body data analysis are provided in the SI.

For the NA-AIMD trajectory computations, we have generated 100 random configurations of Methanol (geometries and velocities) from a 300K AIMD trajectory of neutral methanol calculated at the CASSCF level using the MOLCAS package ${ }^{46}$ at the $(12 \mathrm{e}, 10 \mathrm{o}) /$ aug- $^{-}$ cc-pVTZ active space / basis-set level. Each of the 100 configurations was used for initiating a non-adiabatic molecular dynamics calculation of the methanol dication in each one of the seven lowest-lying dicationic electronic excited states. This generated 700 trajectories altogether. The ensuing non-adiabatic dynamics was approximated using surface-hopping molecular dynamics trajectories ${ }^{47}$ generated at the XMS-CASPT2/(8e,8o)/aug-cc-pVDZ/densityfitting level using the BAGEL electronic structure package ${ }^{29}$ within the so-called "SS-SR" contraction scheme ${ }^{48}$ used for internally contracted basis functions in CASPT2 where a vertical shift set to $0.2 E_{h}$ was used. The BAGEL code was interfaced with a modified version Newton-X (v1.4.0) program ${ }^{49}$ for carrying out the surface hopping dynamics ${ }^{28}$. To facilitate the trajectory calculations, the system in adiabatic state $n$ is allowed to hop only to the state $m$ nearest in energy above or below it (i.e. we neglect the non-adiabatic coupling terms $\tau_{n m}$ unless $|n-m|=1$ ). We modified the way Newton-X interfaces with the BAGEL code to enable this approximation. The time step for the NA-AIMD trajectories is $0.3 \mathrm{fs}$. The ab initio dynamics are followed for at most 300 fs or until the inter-fragment velocities are observed to reach an asymptotic monotonic behavior. At this stage, the effect of the residual long-range Coulomb repulsion on the final velocities is taken into account using the classical 
equations of motion applied to the center of masses of the resulting cationic fragments.

\section{Supporting Information}

- Detailed description of the three-body data analysis proceedures as well as the computational methods

- Selected non-adiabatic AIMD trajectory movies, showing the different CE mechanisms described in the manuscripts

\section{Acknowledgements}

We acknowledge funding from the ERC grant \#306783 and ISF grants \#1369/17 189/14 as well as funding from the Wolfson foundation. EL and RB gratefully thank Drs. Toru Shiozaki (Northwestern University) and Igor Schapiro (Hebrew University) for their advice and help in setting up and using the BAGEL code.

\section{References}

(1) Kukk, E.; Motomura, K.; Fukuzawa, H.; Nagaya, K.; Ueda, K. Molecular Dynamics of XFEL-Induced Photo-Dissociation, Revealed by Ion-Ion Coincidence Measurements. Appl. Sci. 2017, 7, 531.

(2) Erk, B. et al. Imaging charge transfer in iodomethane upon x-ray photoabsorption. Science 2014, 345, 288-291.

(3) Takanashi, T. et al. Ultrafast Coulomb explosion of a diiodomethane molecule induced by an X-ray free-electron laser pulse. Phys. Chem. Chem. Phys. 2017, 19, 19707-19721.

(4) Vager, Z.; Naaman, R.; Kanter, E. P. Coulomb Explosion Imaging of Small Molecules. Science 1989, 244, 426-431. 
(5) Strasser, D.; Lammich, L.; Krohn, S.; Lange, M.; Kreckel, H.; Levin, J.; Schwalm, D.; Vager, Z.; Wester, R.; Wolf, a.; Zajfman, D. Two- and three-body kinematical correlation in the dissociative recombination of H3 +. Phys. Rev. Lett. 2001, 86, 779-782.

(6) Itakura, R.; Liu, P.; Furukawa, Y.; Okino, T.; Yamanouchi, K.; Nakano, H. Two-body Coulomb explosion and hydrogen migration in methanol induced by intense 7 and 21 fs laser pulses. J. Chem. Phys. 2007, $12 \%$.

(7) Xu, H.; Okino, T.; Kudou, T.; Yamanouchi, K.; Roither, S.; Kitzler, M.; Baltuska, A.; Chin, S. L. Effect of laser parameters on ultrafast hydrogen migration in methanol studied by coincidence momentum imaging. J. Phys. Chem. A 2012, 116, 2686-2690.

(8) Posthumus, J. H. The dynamics of small molecules in intense laser fields. Rep. Prog. Phys. 2004, 67, 623-665.

(9) Frasinski, L. J.; Plumridge, J.; Posthumus, J. H.; Codling, K.; Taday, P. F.; Divall, E. J.; Langley, a. J. Counterintuitive alignment of $\mathrm{H} 2+$ in intense femtosecond laser fields. Phys. Rev. Lett. 2001, 86, 2541-2544.

(10) Livshits, E.; Baer, R. Time-dependent density-functional studies of the D2 Coulomb explosion. J. Phys. Chem. A 2006, 110, 8443-8450.

(11) Prabhudesai, V. S.; Lev, U.; Natan, A.; Bruner, B. D.; Diner, A.; Heber, O.; Strasser, D.; Schwalm, D.; Ben-Itzhak, I.; Hua, J. J.; Esry, B. D.; Silberberg, Y.; Zajfman, D. Tracing the photodissociation probability of $\mathrm{H} 2+$ in intense fields using chirped laser pulses. Phys. Rev. A 2010, 81, 2-5.

(12) Ibrahim, H.; Lefebvre, C.; Bandrauk, A. D.; Staudte, A.; Légaré, F. H2: The benchmark molecule for ultrafast science and technologies. J. Phys. B: At., Mol. Opt. Phys. 2018, 51. 
(13) Runge, E.; Gross, E. K. U. Density functional theory for time dependent systems. Phys. Rev. Lett. 1984, 52, 997-1000.

(14) Send, R.; Furche, F. First-order nonadiabatic couplings from time-dependent hybrid density functional response theory: Consistent formalism, implementation, and performance. J. Chem. Phys. 2010, 132, 044107-12.

(15) Zhang, D.; Peng, D.; Zhang, P.; Yang, W. Analytic gradients, geometry optimization and excited state potential energy surfaces from the particle-particle random phase approximation. Phys. Chem. Chem. Phys. 2015, 17, 1025-1038.

(16) Adamowicz, L.; Laidig, W. D.; Bartlett, R. J. Analytical gradients for the coupledcluster method. Int. J. Quantum Chem. 1984, 26, 245-254.

(17) Gauss, J.; Stanton, J. F. Analytic gradients for the coupled-cluster singles, doubles, and triples (CCSDT) model. J. Chem. Phys. 2002, 116, 1773-1782.

(18) Krylov, A. I. Equation-of-motion coupled-cluster methods for open-shell and electronically excited species: The Hitchhiker's guide to Fock space. Annu. Rev. Phys. Chem. 2008, 59, 433-462.

(19) Roos, B. O.; Taylor, P. R.; Sigbahn, P. E. a. A complete active space SCF method (CASSCF) using a density matrix formulated super-CI approach. Chem. Phys. 1980, 48, 157-173.

(20) Roos, B. O.; Linse, P.; Siegbahn, P. E. M.; Blomberg, M. R. A. A Simple Method for the Evaluation of the 2nd-Order Perturbation Energy from External Double-Excitations with a Casscf Reference Wavefunction. Chem. Phys. 1982, 66, 197-207.

(21) Corkum, P. B. Plasma Perspective on Strong-Field Multiphoton Ionization. Phys. Rev. Lett. 1993, r1, 1994-1997. 
(22) Månsson, E. P.; Guénot, D.; Arnold, C. L.; Kroon, D.; Kasper, S.; Dahlström, J. M.; Lindroth, E.; Kheifets, A. S.; Huillier, A. L.; Sorensen, S. L.; Gisselbrecht, M. Double ionization probed on the attosecond timescale. Nat. Phys. 2014, 10, 207-211.

(23) Zhou, X.; Ranitovic, P.; Hogle, C. W.; Eland, J. H.; Kapteyn, H. C.; Murnane, M. M. Probing and controlling non-Born-Oppenheimer dynamics in highly excited molecular ions. Nat. Phys. 2012, 8, 232-237.

(24) Gagnon, E.; Ranitovic, P.; Tong, X.-M. M.; Cocke, C. L.; Murnane, M. M.; Kapteyn, H. C.; Sandhu, A. S. Soft X-ray-Driven Femtosecond Molecular Dynamics. Science 2007, 317, 1374-1378.

(25) Molloy, R. D.; Danielsson, A.; Karlsson, L.; Eland, J. H. Double photoionisation spectra of small molecules and a new empirical rule for double ionisation energies. Chem. Phys. 2007, 335, 49-54.

(26) Luzon, I.; Jagtap, K.; Livshits, E.; Lioubashevski, O.; Baer, R.; Strasser, D. Singlephoton Coulomb explosion of methanol using broad bandwidth ultrafast EUV pulses. Phys. Chem. Chem. Phys. 2017, 19, 13488-13495.

(27) Streeter, Z. L.; Yip, F. L.; Lucchese, R. R.; Gervais, B.; Rescigno, T. N.; McCurdy, C. W. Dissociation dynamics of the water dication following one-photon double ionization. I. Theory. Phys. Rev. A 2018, 98, 053429.

(28) Park, J. W.; Shiozaki, T. On-the-Fly CASPT2 Surface-Hopping Dynamics. J. Chem. Theory Comput. 2017, 13, 3676-3683.

(29) Shiozaki, T. BAGEL: Brilliantly Advanced General Electronic-structure Library. WIREs Comput Mol Sci 2018, 8, e1331.

(30) Ekanayake, N. et al. Mechanisms and time-resolved dynamics for trihydrogen cation $(\mathrm{H} 3+)$ formation from organic molecules in strong laser fields. Sci. Rep. 2017, 7, 4703. 
(31) Ando, T.; Shimamoto, A.; Miura, S.; Iwasaki, A.; Nakai, K.; Yamanouchi, K. Coherent vibrations in methanol cation probed by periodic $\mathrm{H} 3+$ ejection after double ionization. Communications Chemistry 2018, 1, 7.

(32) Eland, J. H. The origin of primary H3+ ions in mass spectra. Rapid Commun. Mass Spectrom. 1996, 10, 1560-1562.

(33) Ekanayake, N. et al. H2 roaming chemistry and the formation of $\mathrm{H} 3+$ from organic molecules in strong laser fields. Nat. Commun. 2018, 9, 5186.

(34) De, S.; Rajput, J.; Roy, A.; Ghosh, P. N.; Safvan, C. P. Formation of H3+ due to intramolecular bond rearrangement in doubly charged methanol. Phys. Rev. Lett. 2006, $97,213201$.

(35) Mebel, A. M.; Bandrauk, A. D. Theoretical study of unimolecular decomposition of allene cations. J. Chem. Phys. 2008, 129, 224311.

(36) Polanyi, M. Zum Ursprung der chemischen Energie. Zeitschrift für Physik 1920, 3, 31-35.

(37) Dalitz, R. CXII. On the analysis of $\tau$-meson data and the nature of the $\tau$-meson. Lond.Edinb.Dubl.Phil.Mag 1953, 44, 1068-1080.

(38) Strasser, D.; Lammich, L.; Kreckel, H.; Krohn, S.; Lange, M.; Naaman, A.; Schwalm, D.; Wolf, A.; Zajfman, D. Breakup dynamics and the isotope effect in $\mathrm{H} 3+$ and D3+ dissociative recombination. Phys. Rev. A 2002, 66, 032719.

(39) Laperle, C. M.; Mann, J. E.; Clements, T. G.; Continetti, R. E. Three-body dissociation dynamics of the low-lying rydberg States of H 3 and D3. Phys. Rev. Lett. 2004, 93, $2-5$.

(40) Bucksbaum, P. H.; Berrah, N. Brighter and faster: The promise and challenge of the x-ray free-electron laser. Phys. Today 2015, 68, 26-32. 
(41) Reedy, D. et al. Dissociation dynamics of the water dication following one-photon double ionization. II. Experiment. Phys. Rev. A 2018, 98, 053430.

(42) newport, solstice ace, spectra-physics. 2014.

(43) Sutherland, J.; Christensen, E.; Powers, N.; Rhynard, S.; Painter, J.; Peatross, J. High harmonic generation in a semi-infinite gas cell. Opt. Express 2004, 12, 4430-4436.

(44) Salieres, P.; Ditmire, T.; Budil, K. S.; Perry, M. D.; L'Huillier, A. Spatial profiles of high-order harmonics generated by a femtosecond Cr:LiSAF laser. J. Phys. B: At., Mol. Opt. Phys. 1994, 27, L217-L222.

(45) Kandhasamy, D. M.; Albeck, Y.; Jagtap, K.; Strasser, D. 3D Coincidence Imaging Disentangles Intense Field Double Detachment of SF6-. J. Phys. Chem. A 2015, 119, $8076-8082$.

(46) Aquilante, F.; Autschbach, J.; Carlson, R. K.; Chibotaru, L. F.; Delcey, M. G.; De Vico, L.; Fdez. Galván, I.; Ferré, N.; Frutos, L. M.; Gagliardi, L. a. Molcas 8: New capabilities for multiconfigurational quantum chemical calculations across the periodic table. J. Comput. Chem. 2016, 37, 506-541.

(47) Tully, J. C. Molecular-Dynamics with Electronic-Transitions. J. Chem. Phys. 1990, 93, $1061-1071$.

(48) Vlaisavljevich, B.; Shiozaki, T. Nuclear energy gradients for internally contracted complete active space second-order perturbation theory: Multistate extensions. J. Chem. Theory Comput. 2016, 12, 3781-3787.

(49) Barbatti, M.; Ruckenbauer, M.; Plasser, F.; Pittner, J.; Granucci, G.; Persico, M.; Lischka, H. Newton-X: a surface-hopping program for nonadiabatic molecular dynamics. WIREs Comput Mol Sci 2014, 4, 26-33. 